DOI: $10.33947 / 1982-3282-v 15 n 3-4-4374$

\title{
ATIVIDADE ANTIBACTERIANA DOS ÓLEOS ESSENCIAIS DE HORTELÃ PIMENTA (MENTHA PIPERITA), HORTELÃ JAPONESA (MENTHA ARVENSIS) E MANJERICÃO (OCIMUM BASILICUM) FRENTE A CEPAS ATCC DE SALMONELLA ENTERICA E STAPHYLOCOCCUS AUREUS
}

\section{ANTIBACTERIAL ACTIVITY OF THE ESSENTIAL OILS OF PEPPER MINT (MENTHA PIPERITA), JAPANESE MINT (MENTHA ARVENSIS) AND BASIL (OCIMUM BASILICUM) AGAINST SALMONELLA ENTERICA AND STAPHYLOCOCCUS AUREUS ATCC}

\author{
Kelvin Bispo da Silva ${ }^{1}$, Priscila Luiza Mello²
}

Submetido em: 03/12/2020

Aceito em: 28/07/2021

\section{RESUMO}

Introdução: Os óleos essenciais possuem diversas atividades farmacológicas, destacando sua ação antibacteriana, antifúngica, anti-inflamatória e analgésica. O seu uso como terapia alternativa, tem evoluído muito ao longo dos anos. Objetivo: Determinar a atividade antibacteriana dos óleos essenciais de Ocimum basilicum (Manjericão), Mentha piperita (Hortelã pimenta) e Mentha arvensis (Hortelã japonesa) sobre cepas bacterianas ATCC de Staphylococcus aureus e Salmonella enterica. Materiais e métodos: A atividade antibacteriana foi determinada através da técnica de difusão de discos de papel em ágar, utilizando concentrações puras e diluídas dos óleos essenciais. Resultados: A cepa de Staphylococcus aureus se mostrou sensível somente ao óleo de Ocimum basilicum puro, enquanto a Salmonella entérica apresentou sensibilidade a todos os óleos essenciais, com exceção do Ocimum basilicum diluído. Conclusão: A Salmonella enterica apresentou maior sensibilidade aos óleos testados, enquanto os Staphylococcus aureus só foram sensíveis Ocimum basilicum puro. Pode-se dizer que a atividade antimicrobiana dos óleos essenciais indica a presença de moléculas com potencial farmacológico a ser explorado.

DESCRITORES: Óleo essencial; Atividade antibacteriana; Ocimum basilicum Salmonella entérica; Staphylococcus aureus; Mentha piperita; Mentha arvensis

\section{ABSTRACT}

Introduction: Essential oils have several pharmacological activities, highlighting your activity antibacterial, antifungal, anti-inflammatory and analgesic. Its use as therapy alternative, has evolved a lot over the years. Aim: Determine the antibacterial activity of the essential oils of Ocimum basilicum (Basil), Mentha piperita (Peppermint Mint) and Mentha arvensis (Japanese Mint) on bacterial strains of Staphylococcus aureus and Salmonella enterica. Materials and methods: Antibacterial activity was determined through the technique of diffusing paper disks on Agar, using concentrations pure and diluted essential oils. Results: The Staphylococcus aureus strain was sensitive to Ocimum basilicum oil only in pure contraction, the Salmonella enterica strain was sensitive to all essential oils but there was no halo for the diluted Ocimum basilicum oil. Conclusion: Salmonella enterica showed greater sensitivity was more sensitive to the oils tested, whereas Staphylococcus aureus were only sensitive to pure Ocimum basilicum. the antimicrobial activity of essential oils indicates the presence of molecules with pharmacological potential to be explored.

DESCRIPTORS: Essencial oil; Antibacterial activity; Ocimum basilicum; Salmonella entérica; Staphylococcus aureus; Mentha piperita, Mentha arvensis

\footnotetext{
${ }^{1}$ Bacharel em Farmácia pela Universidade Univeritas - UNG. E-mail: kelvin.kbs23@gmail.com

${ }^{2}$ Bióloga, Doutora em Biologia Geral e Aplicada pela Universidade Estadual Paulista Júlio de Mesquita - UNESP. Docente do Curso de Pós-graduação em Enfermagem da Universidade Guarulhos - UNG. E-mail: priscila_mello@msn.com
} 


\section{INTRODUÇÃO}

Espécies vegetais ao longo de sua evolução desenvolveram vias metabólicas que permitem sintetizar uma grande variedade de metabólitos secundários. Entre a diversidade de metabólitos secundários produzidos pelas plantas há compostos de baixo peso molecular, voláteis com aroma característico. Esses são comumente chamados de compostos aromáticos ${ }^{1}$. Os compostos aromáticos podem ser encontrados em diversas partes da planta, tais como flores, botões, folhas, raízes e rizomas ${ }^{2}$.

São acumulados em órgãos secretores, podendo ser internos (idioblastos, canais e bolsas) ou externos (tricomas secretores e osmóforos). Através de técnicas de microscopia são visualizados nesses órgãos, gotículas refrativas com forte odor. As espécies vegetais ao serem submetidas a processos extrativos produzem líquidos oleosos, voláteis de odor característico, sendo chamados de óleos voláteis ou óleos essenciais ${ }^{1-2}$.

Os óleos essenciais além do odor e da volatilidade, possuem outras características marcantes, são solúveis em solventes orgânicos, possuem sabor acre, são incolores ou amarelados, sensíveis a variação de luz, calor, umidade e metais e possuem atividade óptica ${ }^{3}$. O consumo dos óleos essenciais é grande, sendo produzidas entre 45,000 e 50,000 toneladas anualmente. A indústria alimentícia, cosmética e farmacêutica são consumidores desses compostos, principalmente pelas suas propriedades antissépticas, antioxidantes e antirradicais livres. Há um crescente aumento da produção agrícola de plantas que produzem óleos essenciais principalmente para o controle de pragas ${ }^{2}$.

Os óleos essenciais possuem diversas atividades farmacológicas, destacando sua ação antibacteriana, antifúngica, anti-inflamatória, analgésica e efeitos no sistema gastrointestinal ${ }^{4}$. Entre as espécies produtoras de óleos essenciais são destaques as plantas da família Lamiaceae como as espécies do gênero Mentha e o Ocimum basilicum ${ }^{2}$.

O uso de plantas medicinais na forma de extratos, como terapia alternativa, tem evoluído muito ao longo dos anos, garantindo ações benéficas à saúde ${ }^{5}$. O uso de plantas medicinais e de fácil acesso à população, principalmente a que não tem acesso a medicamentos industrializados. Entretanto, o uso sem controle de plantas medicinais e seus produtos, ausentes de testes toxicológicos, ensaios pré-clínicos e clínicos pode ocasionar danos à saúde ${ }^{6}$.

O gênero Mentha ou hortelã trata-se de uma planta herbácea da família Lamiaceae, possuindo uma grande variedade de espécies conhecidas. Sua origem é asiática, mas seu cultivo é comum em todo o mundo. Seu uso principal é como tempero em uma variedade de pratos. Na medicina popular como medicamento fitoterápico é indicado para o tratamento de transtornos intestinais, vômitos, flatulência, enjoo, antisséptico bucal, tratamento de aftas, inflamações de garganta e boca, além de possuir ação calmante principalmente na forma de infusão e óleo essencial sendo rico em mentol e mentona?

O Ocimum basilicum (Manjericão) planta da família Lamiaceae de origem asiática é cultivada em várias partes do mundo 8 .

As cepas de Staphylococcus aureus são uma das principais bactérias responsáveis por causar infecções em hospitais. Conforme implantação da antibioticoterapia essas cepas passaram a adquirir resistência a antibióticos, sendo que em 1970 surgiram cepas resistentes a meticilina ou as chamadas superbactérias, se espalhando rapidamente pelo ambiente, sendo a única forma de tratamento à vancomicina ${ }^{9}$. Enquanto todas as Salmonellas são potencialmente patogênicas. Elas penetram quase sempre pela via oral, através de comida e água contaminada. Entre os fatores que facilitam a infecção por essas bactérias, estão: estado nutricional, imunidade, acidez estomacal e integridade da flora bacteriana. As salmonelas são responsáveis gastroenterite, febre tifoide (causada principalmente por sorotipos Typhi e Paratyphi, sepse (comum em pacientes imunocomprometidos) e meningite . $^{8}$

Tendo em vista os fatores acima citados, o objetivo do presente trabalho foi verificar a atividade antimicrobiana dos óleos essenciais de Mentha piperita (hortelã pimenta), Mentha arvensis (hortelã japonesa) e Ocimum basilicum (manjericão) frente a linhagens de ATCC de Salmonella enterica e Staphylococcus aureus. 


\section{MATERIAIS E MÉTODOS}

\section{1 Óleos essenciais}

Foram testados os óleos essenciais de Hortelã japonesa (Mentha arvensis), Hortelã pimenta (Mentha piperita) e Manjericão (Ocimum basilicum). Os óleos essenciais foram adquiridos em lojas especializadas, sendo o óleo de Manjericão obtido da empresa Florananda Ind. e Com. de cosméticos e produtos naturais LTDA ME - Jau/SP lote 171102 com data de validade 07/2020, óleo essencial de Hortelã pimenta adquirido da empresa Phytotrata Cosméticos Ltda - Guarulhos/SP lote POHP1703 fabricado em 10/2017 com validade 10/2019 e óleo de Hortelã Brasil obtido da empresa M.Z. Cosméticos de LTDA - São Paulo/SP lote 1018047 com validade 10/2021.

\subsection{Microbiologia}

Foram utilizadas linhagens de ATCC de S. aureus (ATCC CCCD-S009) e linhagem de S. enterica (ATCC 6962). O meio de cultura utilizado foi ágar Mueller-Hinton em placas de petri de vidro 100×20 MM, discos de papel estéril sendo 9 discos de $12 \mathrm{~mm}$ e 3 discos de $5 \mathrm{~mm}$, escala de Mc Farland 0,5, solução salina estéril e estufa bacteriológica.

\subsection{Atividade antibacteriana}

A metodologia adotada para verificar a atividade antimicrobiana dos óleos essenciais foi difusão em discos para determinar a sensibilidade das cepas por meio da formação de um halo inibitório. As cepas de $\mathrm{S}$. aureus (ATCC CCCD-S009) e S. enterica (ATCC 6269) foram padronizadas em nível de turbidez na escala de Mc Farland de 0,5. Foram coletadas $0,5 \mathrm{ml}$ das cepas e diluídas em solução salina estéril. Essa solução foi utilizada para inocular em meio de cultura sólido de Mueller - Hinton. Os óleos essenciais de Hortelã pimenta, Hortelã Brasil e Manjericão foram usados em 2 concentrações, sendo uma pura e diluída em solução salina na proporção de $0,5 \mathrm{ml}$ de óleo para $0,5 \mathrm{ml}$ de solução salina estéril. Os óleos essenciais foram impregnados em discos de papel estéril sendo ao todo 6 discos impregnados com $1 \mathrm{ml}$ de óleos na concentração pura e 6 discos impregnado com $1 \mathrm{ml}$ na concentração diluída com solução salina estéril. Os discos de papel com os óleos essenciais foram inoculados no meio solido de Mueller- Hinton para a determinação do halo inibitório. Os meios de cultura foram incubados em estufa bacteriológica durante $24 \mathrm{~h}$ em temperatura de $37^{\circ} \mathrm{C}$.

\section{RESULTADOS}

A tabela 1 indica os resultados obtidos através da formação de halos de inibição dos óleos essenciais de Hortelã japonesa (Mentha arvensis), Hortelã pimenta (Mentha piperita) e Manjericão (Ocimum basilicum) e sua atividade contra as cepas de Staphylococcus aureus (ATCC CCCD-S009) e Salmonella enterica (ATCC 6269) nas concentrações pura e diluída.

Tabela 1. Valores dos halos de inibição (em $\mathrm{mm}$ ) dos óleos essenciais frente as cepas ATCC de S. aureus e S. enterica nas concentrações puras e diluídas.

\begin{tabular}{cccc}
\hline Óleo essencial & Concentração & $\begin{array}{c}\text { S. aureus } \\
\text { ATCC CCCD-S009 }\end{array}$ & $\begin{array}{c}\text { S. enterica } \\
\text { ATCC 6269 }\end{array}$ \\
\hline $\begin{array}{c}\text { Mentha arvensis } \\
\text { (Hortelã japonesa) }\end{array}$ & Puro & $0 \mathrm{~mm}$ & $16 \mathrm{~mm}$ \\
\cline { 2 - 4 } & $1: 2$ & $0 \mathrm{~mm}$ & $13 \mathrm{~mm}$ \\
\hline $\begin{array}{c}\text { Mentha piperita } \\
\text { (Hortelã pimenta) }\end{array}$ & Puro & $0 \mathrm{~mm}$ & $14 \mathrm{~mm}$ \\
\cline { 2 - 4 } & $1: 2$ & $0 \mathrm{~mm}$ & $13 \mathrm{~mm}$ \\
\hline $\begin{array}{c}\text { Ocimum basilicum } \\
\text { (Manjericão) }\end{array}$ & Puro & $14 \mathrm{~mm}$ & $16 \mathrm{~mm}$ \\
\cline { 2 - 4 } & $1: 2$ & $0 \mathrm{~mm}$ & $0 \mathrm{~mm}$ \\
\hline
\end{tabular}

DOI: $10.33947 / 1982-3282-v 15 n 3-4-4374$ 
Nos testes de sensibilidade frente a cepa de S. aureus só obtivemos atividade inibitória com o óleo essencial de Manjericão (Ocimum basilicum) na concentração pura, cujo halo foi de $14 \mathrm{~mm}$. Os óleos essenciais de Hortelã pimenta e Hortelã japonesa não apresentaram atividade antibacteriana em nenhuma concentração testada.

Já os testes de sensibilidade contra cepas de $\mathrm{S}$. enterica apresentaram mais promissores em ambas as concentrações. No teste de sensibilidade o óleo de Manjericão (Ocimum basilicum apresentou halo de 16mm frente a S. entérica na concentração pura. Já a Hortelã pimenta (Mentha piperita) apresentou halo inibitório tanto na concentração pura como na diluída 1:2 para a mesma cepa, sendo halos de 14mm e 13mm respectivamente. Para Hortelã japonesa (Mentha arvensis) os halos foram 16mm na concentração pura e13mm na concentração diluída 1:2.

\section{DISCUSSÃO}

No presente trabalho, ambas cepas ATCCs foram escolhidas por possuírem característica que permitam ser utilizadas para testes de suscetibilidade. Em uma comparação entre os óleos testados, o manjericão foi o único que apresentou atividade contra as duas cepas, entretanto ao ser diluído o óleo não possui mais atividade antibacteriana. A atividade antibacteriana do óleo de manjericão pode ser devido a presença do linalol ${ }^{10}$. Pesquisas mostram que o linalol é um dos ativos mais efetivos contra bactérias ${ }^{11}$.

Em testes de sensibilidade utilizando o óleo essencial de Mentha piperita através do método de difusão em poços de ágar se mostrou efetivo contra cepas de bactérias gram negativas e gram positivas, sendo as cepas de bactérias gram positivas Staphylococcus aureus e Streptococcus pyogenes, as cepas gram negativas Escherichia coli e Klebsiella pneumoniae. As duas cepas bacterianas gram positivas Staphylococcus aureus e Streptococcus pyogenes foram sensíveis ao óleo essencial com a zona de inibição 17,2 e 13,1 mm, respectivamente. A zona de inibição para bactérias gram negativas varia de 5,1 a $12,4 \mathrm{~mm}^{12}$. As bactérias Gram negativas se mostraram mais resistentes ao óleo de Mentha piperita, a resistência pode ser explicada devido a presença de lipopolissacarideos presentes na membrana externa das bactérias gram negativas protegendo a membrana do ataque químico dos óleos essenciais ${ }^{13}$.

Através da concentração inibitória mínima em concentrações de até $2 \%$ mostrou ação contra cepas de Erwinia carotovora, encontradas em alfaces, mostrou halos inibitório que variam de 24 a $28 \mathrm{~mm}$. Contra cepas de Staphylococcus aureus e Streptococcus mutans o óleo de manjericão atividade bacteriostática nas considerações de 36,0 $\mathrm{mg} / \mathrm{ml}$. No estudo foi testada a capacidade bactericida do óleo de manjericão, o mesmo se mostrou ativo contra as cepas de Staphylococcus aureus ${ }^{14}$.

A Mentha arvensis (Hortelã japonesa) cujo competente e ativo principal é o mentol. Em testes realizados em Staphylococcus aureus resistentes a meticilina, o óleo essencial extraído por solventes orgânicos etanoicos foi associado a aminoglicosídeos para potencializar seus efeitos contra cepas resistentes. O óleo associado com gentamicina se mostrou mais eficiente ${ }^{15}$.

Estudos apontam que bactérias gram positivas são mais sensíveis aos óleos essenciais, do que as bactérias gram negativas ${ }^{2,4}$. A explicação para esse fato está na complexidade da parede celular das bactérias gram negativas, a presença de uma membrana externa e lipopolissacarídeo, dificulta a difusão, disseminação e consequentemente a ação antibacteriana ${ }^{3,6,7,10}$.

Para que os óleos essenciais desempenhem atividade antimicrobiana deve ser levado em conta diversos fatores, composição química deles, estocagem, impurezas presentes nos óleos, tipo dos microrganismos testados e condições onde os óleos foram estocados, esses fatores podem desencadear uma diminuição da atividade antimicrobiana16. Portanto, há uma diversidade de fatores que interferem na produção de óleos essenciais, podendo destacar a qualidade do solo, quantidade de nutrientes e água; fatores climáticos, quantidade de radiação solar, temperatura, época do ano. Como os óleos essenciais são metabolitos secundários utilizados pelas espécies vegetais para a proteção é necessário que a planta tenha estímulos agressivos para a produção de componentes específicos de defesa dos óleos e épocas de reprodução. Todos esses fatores devem ser levados em conta quando se testa a atividade antibacteriana dos óleos essenciais ${ }^{17}$.

Existem diversos métodos na literatura com intenção de determinar a atividade antimicrobiana dos óleos essenciais. Os diferentes métodos descritos na literatura mostram resultados diferentes, alguns mais efetivos que

DOI: 10.33947/1982-3282-v15n3-4-4374 
outros. A falta de padronização de ensaios na farmacopeia impossibilita a comparação entre os resultados. Portanto, os resultados demonstraram que esses óleos podem ser usados como método prévio, devido ser reconhecido por determinar a sensibilidade de bactérias, produzindo resultados qualitativos e semiquantitativos.

\section{CONCLUSÃO}

Os óleos essenciais utilizados neste trabalho, demonstraram uma atividade antibacteriana em potencial. O óleo essencial de manjericão é ativo contra as duas cepas, entretanto apenas na concentração pura. A cepa de Staphylococcus aureus se mostrou mais resistente a ação dos óleos essenciais de hortelã. A resistência pode ser explicada por possível desvio de qualidade dos óleos, presença de impurezas ou necessidade de uma quantidade mais elevada de óleo essencial.

As cepas de Salmonella enterica mostraram ser mais sensíveis aos óleos testados, essa sensibilidade pode ser útil na terapêutica contra salmonelose causadas por ingestão de água ou alimentos contaminados presente em países em desenvolvimento. O uso de óleos essenciais abre uma perspectiva a respeito de uma terapia antibacteriana eficaz e de baixo custo contra infecções causadas por bactérias e outros microrganismos.

\section{REFERÊNCIAS BIBLIOGRÁFICAS}

1. Cunha AP, da Graça JAB. Farmacognosia e fitoquímica. 2. ed. Lisboa: Fundação Calouste Gulbenkian; 2009. 674 p.

2. Araújo LS, Araújo RS, Serra JL, Nascimento AR. Composição química e susceptibilidade do óleo essencial de orégano (Origanum vulgare L., família Lamiaceae) frente às cepas de Escherichia coli, Staphylococcus aureus e Salmonella choleraesuis. Boletim do Centro de Pesquisa de Processamento de Alimentos. 2015;33(1).

3. Simões CMO, Schenkel EP, Gosmann G, Melo JCP, Mentz LA, Petrovick, P. R. Farmacognosia: da planta ao medicamento. 6 (th) ed. Florianópolis: UFSC; 2010.

4. Barbosa LN, da Silva Probst I, Andrade BFMT, Alves FCB, Albano M, de Souza MDLR, Júnior AF. Propriedades antibacterianas e químicas in vitro de óleos essenciais, incluindo plantas nativas do Brasil, contra bactérias patogênicas e resistentes. Journal of oleo Science. 2015;64(3):289-298.

5. Lorenzi H, Matos FJDA. Plantas medicinais no Brasil: nativas e exóticas. 2. ed. Nova Odessa: Instituto Plantarum de Estudos da Flora Ltda; 2008.

6. Veiga Junior VF, Pinto AC, Maciel, M. A. M. Plantas medicinais: cura segura?. Química nova. 2005;28(3):519-528.

7. Costa CMGR, Santos MS, Barros HMM, Agra PFM, Farias MAA. Efeito inibitório do óleo essencial de manjericão sobre o crescimento in vitro de Erwinia carotovora. Tecnologia \& Ciência Agropecuária. 2009;3(3):35-38.

8. Martins AGLDA, Nascimento AR, Mouchrek Filho JE, Mendes Filho NE, Souza AG, Aragão NE, Silva, DSVD. Atividade antibacteriana do óleo essencial do manjericão frente a sorogrupos de Escherichia coli enteropatogênica isolados de alfaces. Ciência Rural. 2010;40(8):1791-1796.

9. Santos ALD, Santos DO, Freitas CCD, Ferreira BLA, Afonso IF, Rodrigues CR, Castro HC Staphylococcus aureus: visitando uma cepa de importância hospitalar. Jornal Brasileiro de Patologia e Medicina Laboratorial. 2007;43(6):413423.

10. Pattnaik S, Subramanyam VR, Bapaji M, Kole CR. Antibacterial and antifungal activity of aromatic constituents of DOI: 10.33947/1982-3282-v15n3-4-4374 
essential oils. Microbios. 1997;89(358):39-46.

11. Santos CHS, Piccoli RH, Tebaldi VMR. Atividade antimicrobiana de óleos essenciais e compostos isolados frente aos agentes patogênicos de origem clínica e alimentar. Revista do Instituto Adolfo Lutz. 2017;1719(76):1-8.

12. Singh R, Shushni MA, Belkheir A. Atividades antibacterianas e antioxidantes de Mentha piperita L. Arabian Journal of Chemistry. 2015;8(3):322-328.

13. İşcan G, Kirimer N, Kürkcüoğlu M, Başer HC, DEMIrci F. Triagem antimicrobiana de óleos essenciais de Mentha piperita. Jornal de química agrícola e de alimentos. 2002;50(14):3943-3946.

14. Freire ICM, Pérez ALAL, Cardoso AMR, Mariz BALA, Almeida LFD, Cavalcanti YW, Padilha WWN. (2014). Atividade antibacteriana de Óleos Essenciais sobre Streptococcus mutans e Staphylococcus aureus. Revista Brasileira de Plantas Medicinais. 2002;16(2):372-377.

15. Coutinho HD, Costa JG, Lima EO, Falcao-Silva VS, Siqueira-Junior JP. Potentiating effect of Mentha arvensis and chlorpromazine in the resistance to aminoglycosides of methicillin-resistant Staphylococcus aureus. in vivo. 2009;23(2):287-289.

16. Marino M, Bersani C, Comi G. Impedance measurements to study the antimicrobial activity of essential oils from Lamiaceae and Compositae. International journal of food microbiology. 2001;67(3):187-195.

17. Gobbo-Neto L, Lopes NP. Plantas medicinais: Fatores de influência no conteúdo de metabólitos secundários. Química nova. 2007;30(2):374-381. 